\title{
Sports Monitoring with Moving Aerial Cameras Maybe Cost Efficient For Injury Prevention
}

\author{
Deborah Joy Hilton ${ }^{a} b^{*}$ \\ ${ }^{a}$ Deborah Hilton Statistics Online, Australia. \\ ${ }^{b}$ Master of Public Health, Bachelor of Physiotherapy, University of Queensland, Australia.
}

Received 13 March 2020; Accepted 08 July 2020

\begin{abstract}
Objectives: An Australian access economics report (2009) estimated the lifetime cost of care is 5.0 million for a person whom suffers paraplegia and 9.5 million for quadriplegia, and costs/year are approximately $\$ 90,000$. Hilton (2018) on drones at sporting venues discusses their potential to revolutionize injury surveillance monitoring via expert exposure gained for recording, investigation, tracking and monitoring of sporting injuries. Hilton (2018) reviewed rugby union and league Australian spinal cord injury datasets, finding more incident cases in the union then league [1]. Methods/Analysis: Wikipedia reports 20 professional rugby union and 26 rugby league playing fields in Australia. The Australian Institute of Health and Welfare document; Australian sports injury hospitalizations 2011-12 report just under 800 head and neck injuries requiring hospitalization related to rugby-related sports " 35 neck fractures and 348 head fractures". Brisbane's leading drone aerial photography service "Droneworxs" according to previous enquiries by the author charge $\$ 650 /$ hour to monitor a sporting event. A crude drone implementation cost estimate, hypothetically is to utilize this device across 46 professional clubs X 52 weeks one hour/week $=\$ 1,554,800$. A basic hypothetical mathematical cost benefit comparison was performed. Findings: Droneworxs cost divided by healthcare costs/case/year $(\$ 90,000)=17$ so if these injuries are prevented then cost equivalence is reached figurately speaking, then cost benefits accrue. Novelty /Improvement: Drones are not overly expensive compared to spinal cord injury costs. The occasional presence of aerial cameras at sporting venues may also deter repeated foul play, in the same way that webcam cameras deter potential thieves.
\end{abstract}

Keywords: Spinal Cord Injuries; Football; Hospitalised Injuries; Aerial Cameras; Sports Monitoring.

\section{Introduction}

Hilton (2018) studied on moving aerial cameras at sporting venues and whether they have the potential to revolutionize injury surveillance monitoring [1]. These devices have the capacity to produce expert exposure for the purposes of recording, investigation, tracking, and monitoring of sporting injuries.

Unmanned aerial vehicles, also referred to as drones, or flying robots, are controlled from the ground via a remote control or via an onboard computer allowing communication. There is a long history of their usage, which includes being used for military purposes such as peacekeeping or surveillance, agriculture, aerial drops, commercial usage, scientific purposes, data collection, research, surveying, policing, smuggling, rescues, and recreational usage such as aerial photography or drone racing. There is scope for drones to be further potentially utilized in a greater capacity in terms of monitoring sporting events not only to film, but for this film exposure to be further utilized for the purposes of studying causal and precipitating factors associated with injury, for surveillance, and for recording a log of sporting injuries.

\section{*Corresponding author: deborah.hilton@gmail.com \\ do) http://dx.doi.org/10.28991/SciMedJ-2020-0203-3}

$>$ This is an open access article under the CC-BY license (https://creativecommons.org/licenses/by/4.0/).

(C) Authors retain all copyrights. 
Injury surveillance is important for not only providing epidemiological information, but to protect an athlete's health, and for monitoring time trend changes in the frequency and events surrounding injury occurrences. There are countless examples of injury surveillance and tracking databases. Various example include; the National Collegiate Athletic Association Injury Surveillance Program and High School Reporting Information Online with this publication reporting on the first decade of data collection [2], the sport's injury registry of the University Hospital of Lausanne, Switzerland where there were over 3114 recorded reports [3], a 15 year Level I trauma department registry [4], and a 10 year (2005-2015) Victorian database of sport and recreation injuries resulting in major trauma or death [5].

The potential and possibility for more elaborate injury analysis systems may develop and appear in the future due to the emerging use of moving aerial cameras (drones). The Winter Olympics in Sochi (2014) utilized drones for filming ski and snowboarding events and the Atlantic times states that drones are the future of sports broadcasting [6]. Skydio launched a smart, autonomous camera drone (RL) in February 2018 [7], a camera that can maneuver, track and avoid obstacles while also filming. As an example, this camera hence can film a mountain biker while they weave in and out of a forest, dodging trees, and other obstacles.

The computer systems, algorithms and technology that enable coordination, programming and operation of these complex devices is becoming more sophisticated and state of the art. Various authors have delved into the complexities and detail of programming and set up. Khan et al. [8] studied on aerial camera networks for observing moving targets. The algorithms described by this author, allow cooperative motion planning of these aerial cameras in three-dimensional environments to detect and watch numerous targets moving on the ground and at varying elevations. The quality of view is maximized by organizing the zoom, resolution and field of view, making sure that cameras don't collide and allowing them to share the workload of assignment locations. Yadav et al. [9] present a simple, economical and less time-consuming solution using an object tracking algorithm implemented via an Unmanned Aerial Vehicle. The images are obtained from the drone while the moving ground object is detected by colour segmentation techniques. Open Source Computer Vision libraries process the images controlled by an environment created using Robot Operating System (ROS).

Hernandez-Hernandez (2017) worked on how to keep a moving target within the field of view of a Drone's onboard camera via stochastic estimation. They discuss how there is increasing use of drones to capture video in scenarios that also include sports. They describe how the images are combined with a stochastic estimator of the target states, based on the Unscented Kalman Filter. They validate the system comparing results with human pilots with improved statistical results $(96.6 \%$ compared with $83.3 \%$ ). This means that aerial moving cameras potentially can be utilized where the target is kept within the field of view, so that would include sports [10].

Tanaka et al. (2018) explains the real-time image processing framework with an aerial overhead camera for sports [11]. They describe a drone unit with an infrared camera and a small PC for real-time image processing, including surface and object detection. Novel infrared markers are used for robust detection of the four corners of a rectangle and the objects within. They describe how this can be used for an interactive sports coaching application whereby an overhead drone camera unit films a large playing field.

Various sporting events have utilised moving aerial cameras as has been previously mentioned, but in addition the $\mathrm{X}$ Games which is an annual extreme sports event and the Norseman Triathlon which is conducted annually have produced some epic race videos using drone technology [12].

Film currently obtained for sports broadcasting coverage, potentially can be utilized for enhanced viewing and image capture of sporting accidents and injuries. Potentially the film can be zoomed in on, re-wound, re-played and paused, in order to enhance the analysis of injury events. Historically expert witness reports were obtained but these can lack credibility due to poor memory, lack of observation, incorrect interpretation or poor visibility due to being at a significant distance from the action point. Video capture has the ability to overcome some of these obstacles, with the capacity to create an expert eyewitness account.

\subsection{Rugby Union and Rugby League - An Example}

Hilton (2009) and (2018) studied on a look at the past and future expectations [13, 14]. The reviews identified Australian datasets that reported on catastrophic Spinal Cord Injuries (SCI) in Rugby Union (RU) and Rugby League (RL) that were identified from claims data, Australian SCI register data and spinal unit data. The odds ratios or rate ratios as reported in the researches were collated and tabulated so that comparisons between studies were easily viewed with the ratio being considered that of (RU/RL). There are six publications were identified and the ratio ranged from 1.3 to 4.0 with more incident cases of SCI in RU compared to RL, which is a higher rate in RU as compared to RL.

The yearly incremental costs and cumulative lifetime costs of caring for a person whom has sustained a SCI are truly exorbitant. In addition to these excessive costs, is the fact that insurance cover is often insufficient to cover the high cost of care. Estimates from Yeo in the late 1990s as presented by Berry et al. (2006) on SCI in RU and RL, give 
an approximation being the lifetime cost of care for a person with paraplegia as $\$ 1$ million, and that this increases to a staggering \$5 million for a person with tetraplegia [15]. A more recent report, gives alarmingly higher dollar amounts. This Australian access economics report (2009) estimated the lifetime cost of care is 5.0 million for a person whom suffers paraplegia and 9.5 million for a person whom sustains quadriplegia, and costs/year are approximately $\$ 90,000$ for a person whom sustains a spinal cord injury [16].

\section{Methods}

Hilton (2019) presented a crude cost benefit analysis, which is purely hypothetical based on very fuzzy figures and theoretical scenarios [17]. According to Wikipedia, the number of professional RU and RL playing fields within Australia is estimated to be RU [20] and RL [26] and NRL club venues and occasional venues [18, 19].

Droneworxs is one of Brisbane's leading drone aerial photography services [20]. Prior to submission of the abstract to the APACPH conference, Hilton enquired about the hire cost from Droneworxs and was informed that hire cost was at that time $\$ 650 /$ hour to monitor a sporting event. As mentioned, this is a hypothetical mathematical example utilizing this cost estimate figure. This is not a real-life scenario that has been carried out, tested, trialed nor implemented. It is purely a hypothetical example and these costs may have also increased since this initial enquiry was done.

The Australian Institute of Health \& Welfare document entitled; Australian sports injury hospitalizations 2011-12 report just under 800 head and neck injuries requiring hospitalization related to rugby-related sports [21]. More specifically this included 35 neck fractures and 348 head fractures. This information is for reference only and not included in the calculations but gives the reader an idea about the frequency of fractures relating to that body region. The total cost equates to multiplying the number of cases $x$ cost of care / case.

To crudely estimate the cost of implementation of a drone, the hypothetical example is to utilize this device across the 46 professional clubs x 52 weeks one hour /week and this equates to $=\$ 1,554,800$.

\section{Results and Discussion}

Droneworxs cost $(\$ 1,554,800)$ divided by healthcare costs/case/year (cost of caring for a person with paraplegia) $(\$ 90,000)$ equates to 17 so if this number of injuries are prevented then cost equivalence is reached in broad general terminology figurately speaking, after which cost benefits accrue.

There are approximately double this number of neck fractures occurring in rugby related sports / year according to the official document mentioned above. These injuries can occur on professional or amateur playing fields. The cost to implement a drone on every playing field would be exorbitant and it is not feasible to calculate as not all amateur playing fields would have the infrastructure or ability to allow and set this equipment up. Hence the cost of the drone as specified is an estimate only. Given these injuries may occur on amateur playing fields also, the cost benefit equation given above is very theoretical and in practical terms could be highly inaccurate.

As previously stated, this is an extremely fuzzy logic using a hypothetical analysis/ comparison based on scant information which lacks some major details. Hence there are significant flaws in assumption as will be mentioned in the discussion.

The major stumbling block with this crude estimation of costs and possible savings is that this hypothetical scenario fails to consider that SCI do not only occur on professional playing fields so that to only monitor professional playing fields is a somewhat false argument in terms of prevention likelihood in that junior sporting clubs, local ovals and school play grounds can also be places where serious accidents and injuries may take place.

It is impossible to weed out where and when each accident occurs and hence the costs of monitoring with a drone for that specific playing field is difficult to quantify with any degree of accuracy. The hypothetical example is purely given using professional playing fields as an input variable as in fact drone usage at these venues maybe more likely and more feasible to implement as camera footage is currently collected for other purposes. Set up at amateur venues is not as technically feasible as the infrastructure is not normally in place for static cameras so the obstacles are somewhat greater in foreseeing costs which become almost impossible to compute.

Static cameras \& go pro filming is used worldwide and is currently in place for monitoring; rugby league matches, Norwegian male professional football, football, world cup alpine skiing, freestyle ski cross, snowboarding, cyclists, lacrosse, and boxing. Future plans for moving aerial cameras must consider current rules and regulations that relate to these static cameras and go pros [1].

Drones have also been used to film Formula One races, the X Games, the AMA Supercross Series, football, surfing, snowboarding, skateboarding, soccer, and cricket [22]. The National football league have CableCam systems which is a computer-controlled and cable-suspended camera system that maneuverers over the playing field. 
No studies were located on the cost benefits of moving aerial cameras for monitoring sports when Pubmed, PEDro or a general yahoo search was done using search terms; 'moving aerial cameras AND sports'. However, in the SUAS news that discusses the use of cablecam devices, they state that despite this apparatus having good reliability, they take a long time to set up and are expensive. They contrast this with drones, that are easier to set up, and are far more affordable. While no costs and benefits are detailed in dollar figures whether this be real dollars or estimations, they do state that broadly overall drones are more cost effective. Aviation Pros, in a report entitled; Airport benefits of drone technology [23], they mention cost benefit considerations in relation to drones at airports. While this is not drones for monitoring sports events, they state that while the cost of purchase of a drone maybe a fairly steep price point, in that this can range from $\$ 1,500$ to $\$ 20,000$ it should also be considered in comparison to renting a helicopter with a drone onboard, which may vary from $\$ 630-800 /$ hour for a Robinson R44 while a Bell Jetranger is $\$ 1000$ for 40 minutes.

Filming for viewer satisfaction, or live streaming of sports already occurs and if the primary purpose is for viewer satisfaction, then documents and agreements would need to be formalized before a third party has access to the film if it was for an alternative purpose such as analyzing injury mechanisms, that may include re-playing, re-watching, pausing and viewing film in slow motion. These legal and ethical considerations are considerable but necessary before alternative usage of film is allowed. These formalities also involve resources, planning, costs and management.

In addition to sports grounds giving consent for alternative usage of the film obtained that was primarily for sports coverage, there are other regulations that may prevent or delay usage of drones. Currently in America, the Federal Aviation Administration, have rules that mean even despite drones being able to take interesting photographs from unique vantage points, the rules mean that it is difficult to deploy drones in stadiums, arenas, and other venues [22]. However, some football and baseball teams have utilized drones to gather footage to analyze hand placement, foot placement, and spacing between players, for tactical analysis, training and feedback purposes. Again, while a formal cost benefit analysis has not been performed to quantify in detail dollar savings, these clubs view drones as a costefficient and practical alternative to cable cams.

In addition to these formal agreements, the computer system and technical requirements are challenging, in terms of the necessary hardware, software and system requirements which require expert computer system advice and management. Functionality and versatility are other considerations of any computer or technical set up. In addition to the drone or camera cost there maybe other technical requirements for downloading, saving and distributing film images. Difficulties that potentially maybe encountered with technology can include over usage and over expenditure. Costs incurred are also often passed onto the consumers which in this case maybe the spectators, resulting in higher ticket prices and for some people that means sporting events at venues become prohibitive due to cost and hence some members of the public will oppose introduction of drones for this reason.

Moving aerial cameras have complexities related to the computer system requirements not apparent with static cameras or go pros. Natalizio et al. (2013) discussed the complexities of the parameters included in algorithms that are necessary to program fleets of drones [24]. Dynamic vehicle routing plans are formulated. Drones are required to film the point of action, requiring the minimum distance of travel by the drone. The nearest neighbor technique requires the closest flying robot to be deployed. Flying robots can also constantly detect the ball and its location, while also being able to estimate ball trajectories. Ball movement interception is one term that encompasses the fact that drones can be programed to know the point of previous action, and where the next expected action will take place. Preparing these algorithms is complex, with various parameters requiring input into the complex equation.

The legalities also must consider privacy, air space and these legal considerations are complex, costly and challenging. Schneider discusses the disturbing imposition and threat to privacy that may result from drones [25]. Participants may refuse or retaliate against being filmed, while others maybe happy for recordings to be made so hence setting up systems and implementation of plans poses challenges and potential obstacles. In addition, any consideration of the usage of drones must also adhere to current guidelines and protocols. One such document that must be followed is that of the Australian Government Civil Aviation Safety Authority whom publishes drone rules related to safe flying, distance from objects, people, airports and when a person can fly a drone (Australian Government Civil Aviation Safety Authority 2020) [26].

Droneworxs cost was used for the hypothetical mathematical example given, but other companies with this cuttingedge technology also exist. Falkor Systems [27], use innovative object tracking technology to film ski jumps (half pipe) and BASE (Building, Antenna, Span, and Earth Jump) as examples. The cameras allow up close shots, with camera angles unimaginable otherwise.

Squadrone systems has a camera equipped drone that can capture aerial images of a person participating in events such as skiing or snowboarding, made possible through embedded intelligence and mobile apps. The Hexo+ is a selfflying camera, being a product of this modern technology that incorporates smart phone usage, data fusion and complex algorithms [28]. 


\section{Conclusion}

The logistics of setting up operational moving aerial cameras though are complex and many costs need to be factored into the equation. Administrative, legal, management, technology and other infrastructure costs are additional costs to just the drone cost. All parties need to agree on an implementation process and that can become quite complex, along with this being a lengthy convoluted task having all interested persons agree, whether it be managers of sporting clubs, local councils, aviation authorities, spectators, players, sports coaches and officials. Feasibility studies would need to be trialed on a small scale initially to assess benefits and costs. It is also not feasible to install drones at every amateur playing field. While no previous study has looked at specific costs and benefits in terms of injury prevention, several reports make mention that drones are less costly then cablecam apparatus in terms of implementation.

Drones are not overly expensive compared to SCI costs. The occasional presence of aerial cameras at sporting venues may also deter repeated foul play. Not dissimilar in a way that webcams deter intruders, even the presence of false cameras at venues may deter breaching of rugby code rules should a player suspect they are being filmed. Clubs, venues, organizations and authorities may consider that cost of not only the drone, but the admin, legal, technical and unforeseen costs are too high. If you or a member of your family is the person such that footage enables a player to be charged for breach of the rules, then no cost is too extreme or too high. People pay a high price if it means protecting a loved one.

\section{Acknowledgements}

Thank you to Mr. Stephen Hilton for technical expertise and knowledge related to moving aerial cameras (drones).

\section{Declaration of Competing Interest}

The authors declare that they have no known competing financial interests or personal relationships that could have appeared to influence the work reported in this paper.

\section{Ethical Approval}

All procedures performed in studies involving human participants were in accordance with the ethical standards of the Human Research Ethics Committees | NHMRC (Australia) and with the 1964 Helsinki declaration and its later amendments or comparable ethical standards.

\section{References}

[1] Hilton, D. J. (2018). Do Moving Aerial Cameras at Sporting Venues have the Potential to Revolutionize Injury Surveillance Monitoring?. SciFed Journal of Sports Medicine, 1(2).

[2] Kerr, Z. Y., Comstock, R. D., Dompier, T. P., \& Marshall, S. W. (2018). The First Decade of Web-Based Sports Injury Surveillance (2004-2005 through 2013-2014): Methods of the National Collegiate Athletic Association Injury Surveillance Program and High School Reporting Information Online. Journal of Athletic Training, 53(8), 729-737. doi:10.4085/1062-6050 143-17.

[3] Reinberg, A., Reinberg, O., Mechkouri, M., Touitou, Y., \& Smolensky, M. H. (2018). Daily, weekly and annual patterns in children's accidental sport injuries. Chronobiology International, 35(5), 597-616. doi:10.1080/07420528.2018.1459664.

[4] Krutsch, W., Krutsch, V., Hilber, F., Pfeifer, C., Baumann, F., Weber, J., ... Angele, P. (2018). 11.361 sports injuries in a 15year survey of a Level I emergency trauma department reveal different severe injury types in the 6 most common team sports. Sportverletzung · Sportschaden, 32(02), 111-119. doi:10.1055/s-0583-3792.

[5] Ekegren, C. L., Beck, B., Simpson, P. M., \& Gabbe, B. J. (2018). Ten-Year Incidence of Sport and Recreation Injuries Resulting in Major Trauma or Death in Victoria, Australia, 2005-2015. Orthopaedic Journal of Sports Medicine, 6(3), 232596711875750. doi:10.1177/2325967118757502.

[6] Feltman, R. (2014). The Future of Sports Photography: Drones. The Atlantic. Available online: https://www.theatlantic.com/ technology/ archive/2014/02/the-future-of-sports-photography-drones/283896/ (accessed on 1 January 2020).

[7] Ackerman, E. (2018). Skydio announces SDK to make world's cleverest drone even cleverer. IEEE Spectrum. Available online: https://spectrum.ieee.org/automaton/robotics/drones/skydio-announces-sdk-to-make-worlds-cleverest-drone-even-cleverer (accessed on 1 March 2020).

[8] Khan, A., Imran, M., \& Rashid, A. (2018). Aerial Camera Network for Observing Moving Targets. IEEE Sensors Journal, 18(16), 6847-6856. doi:10.1109/jsen.2018.2850856.

[9] Yadav, H., Srivastava, S., Mukherjee, P., \& Lall, B. (2015). A real-time ball trajectory follower using Robot Operating System. 2015 Third International Conference on Image Information Processing (ICIIP). doi:10.1109/iciip.2015.7414826. 
[10] B. Hernandez-Hernandez, J. Martinez-Carranza and J. Rangel-Magdaleno, (2017) Keeping a moving target within the field of view of a Drone's onboard camera via stochastic estimation, Workshop on Research, Education and Development of Unmanned Aerial Systems (RED-UAS), Linkoping, pp. 150-155, doi: 10.1109/RED-UAS.2017.8101659.

[11] Tanaka, K., Tochihara, N., Sato, T., \& Koike, H. (2018). A real-time image processing framework with an aerial overhead camera for sports. Proceedings of the 2018 International Conference on Advanced Visual Interfaces. doi:10.1145/3206505. 3206520 .

[12] DC Rainmaker. Sports, Drones, and Follow-Me Aerial Action Imagery: A State of the Industry. (2015). Available online: https://www.dcrainmaker.com/2015/02/drone-sports-usage.html (accessed on 15 January 2020).

[13] Hilton D. J. (2009) NSW/Australian literature/dataset review comparing rugby union and league spinal injury incidence rate or odds ratios and scrum law sequence changes. Australian Physiotherapy Association Conference, Sports Physiotherapy Australia, Sydney.

[14] Hilton, D. J. (2018). A Review of Rugby Union and League Australian Spinal Cord Injury Datasets - A Look at the Past and Future Expectations. RA Journal of Applied Research, 4(02), 1399-1406. Available online: http://www.rajournals.in/index.php /rajar/ article/view/98 (accessed on 23 January 2020).

[15] Berry, J. G., Harrison, J. E., Yeo, J. D., Cripps, R. A., \& Stephenson, S. C. R. (2006). Cervical spinal cord injury in rugby union and rugby league: are incidence rates declining in NSW? Australian and New Zealand Journal of Public Health, 30(3), 268-274. doi:10.1111/j.1467-842x.2006.tb00869.x.

[16] Report by Access Economics Pty Limited, Australia. (June 2009). The Victorian Neurotrauma Initiative. The economic cost of spinal cord injury and traumatic brain injury in Australia. Available online: http://www.spinalcure.org.au/pdf/Economic-costof-SCI-and-TBI-in-Au-2009.pdf (accessed on 19 March 2020).

[17] Hilton D. J. (2019). Sports monitoring with flying drones (moving aerial cameras) maybe cost efficient if injuries are prevented, 51st Asia-Pacific Academic Consortium for Public Health Conference 2019, November 21-22, Bangkok.

[18] Rugby union in Australia. (2020), Wikipedia. Available online: https://en.wikipedia.org/wiki/Rugby_union_in_Australia (accessed on 10 May 2020).

[19] Rugby league in Australia. (2020), Wikipedia. Available online: https://en.wikipedia.org/wiki/Rugby_league_in_Australia (accessed on 10 May 2020).

[20] Droneworxs. (2020), Brisbane's Leading Drone Aerial Photography Services. Available online: https://droneworxs.com.au/ (accessed on 8 May 2020).

[21] Kreisfeld, R., Harrison, J. E., \& Pointer, S. C. (2014). Australian Sports Injury Hospitalisations: 2011-2012. Canberra, ACT: Australian Institute of Health and Welfare.

[22] Liz Pekler. (2016) SUAS news. The Future of Drones in Live Sports Coverage and Sports Performance Analysis. Available online: https://www.suasnews.com/2016/03/42568/ (accessed on 25 March 2020).

[23] Guillot, B., Dowell, M. (2019). Airport benefits of Drone Technology (UAS technology transforms from threat to valuable tool for improving airport operations). Available online: https://www.aviationpros.com/aircraft/unmanned/article/ 12436848/airport-benefits-of-drone-technology (accessed on 25 March 2020).

[24] Natalizio, E., Surace, R., Loscri, V., Guerriero, F., \& Melodia, T. (2013). Two Families of Algorithms to Film Sport Events with Flying Robots. 2013 IEEE 10th International Conference on Mobile Ad-Hoc and Sensor Systems. doi:10.1109/mass.2013.40.

[25] Schneider, D. (2014) Flying Selfie Bots: Tag-Along Video Drones are here. IEEE Spectrum Available online: https://spectrum. ieee.org/aerospace/aviation/flying-selfie-bots-tagalong-video-drones-are-here (accessed on 11 May 2020).

[26] Australian Government Civil Aviation Safety Authority, Drones, 2020. Available online: https://www.casa.gov.au/drones (accessed on 27 March 2020).

[27] Falkor Systems, (2020). Available online: http://falkorsystems.com/ (accessed on 10 January 2020).

[28] Squadrone Systems, Custom Drones Design and Manufacturing, (2020), Available online: https://squadrone-system.com/en/ (accessed on 18 March 2020). 